\title{
A far-IR and optical 3D view of the starburst driven superwind in NGC 2146
}

\author{
Kathryn Kreckel ${ }^{1}$, Lee Armus ${ }^{2}$, Brent Groves ${ }^{1}$, Mariya Lyubenova $^{3}$, \\ Tanio Diaz-Santos ${ }^{2}$ and Eva Schinnerer ${ }^{1}$ \\ ${ }^{1}$ MPIA ${ }^{2}$ Caltech ${ }^{3}$ Kapteyn Institute
}

Galaxy outflows are a vital mechanism in the regulation of galaxy evolution through feedback and enrichment. NGC 2146, a nearby infrared luminous galaxy (LIRG), presents evidence for outflows along the disk minor axis in all gas phases (ionized, neutral atomic and molecular). We present new far-IR Herschel imaging and spectroscopy of this galaxy from the Key Insights on Nearby Galaxies: a Far-Infrared Survey with Herschel (KINGFISH) project, as well as new optical integral field unit spectroscopy, to map the kinematics and gas excitation in the central $5 \mathrm{kpc}$ and trace the dust distribution (Kreckel et al. 2014). We observe an increased velocity dispersion in the [OI] $62 \mathrm{um}$, [OIII] $88 \mathrm{um}$, [NII] $122 \mathrm{um}$ and [CII] $158 \mathrm{um}$ fine-structure lines that is spatially coincident with shocked gas above and below the disk. Unhampered by extinction, the far-IR lines trace the outflow to the base of the superwind at the disk center, and we discuss the potential for using [CII] as a tracer of outflows such as this in high redshift systems with ALMA. The stellar kinematics are decoupled from the disk rotation seen in all gas phases, which we attribute to a merger that has not produced a fully elliptical morphology.

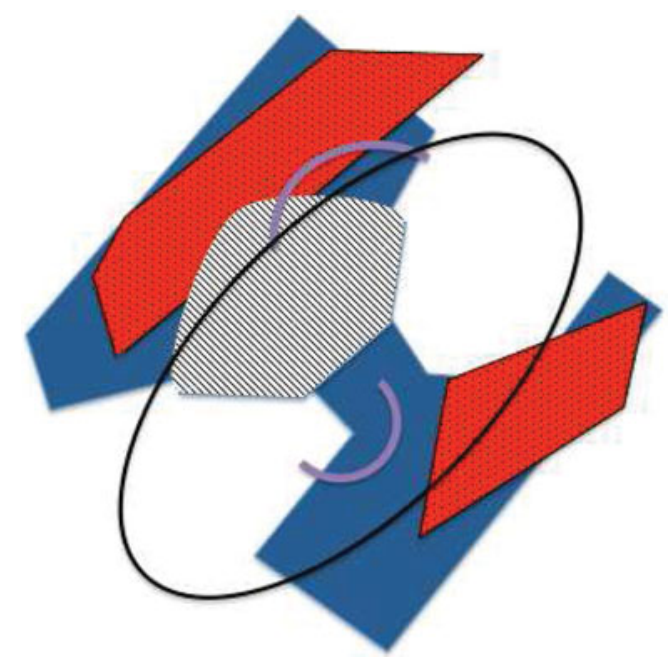

Figure 1. Diagram of outflow indicators in NGC 2146, relative to the stellar disk (black oval). Previous identification of the superwind in soft X-ray emission from hot gas (white hatched) and CO molecular outflows (purple arcs) are consistent with the far-IR [CII] emission from atomic gas in the cone walls (solid blue) and optically identified shock excitation (red dotted). 
A far-IR and optical $3 D$ view of the starburst driven superwind in NGC 2146323

\section{Reference}

Kreckel, K., Armus, L., Groves, B., et al. 2014, ApJ, 790, 26 